\title{
TRANSMISSION OF ARM BASED REAL TIME ECG FOR MONITORING REMOTELY LOCATED PATIENT
}

\author{
Ketaki N. Patil ${ }^{1}$, P. C. Bhaskar ${ }^{2}$ \\ ${ }^{l}$ Mtech Student, Electronics Engineering, Department of Technology, Shivaji University, Maharashtra, India \\ ${ }^{2}$ Professor, Electronics Engineering, Department of Technology, Shivaji University, Maharashtra, India
}

\begin{abstract}
The development of patient monitoring system is of great importance in today's stressed and busy world where people even don't have time to visit doctors again and again. The need of instant medical help is also increasing day by day and can be achieved with wireless technology thereby motivating for wireless patient monitoring system. Also among all the diseases Cardiovascular is one that accounts for high mortality rate across the globe. So, the proposed system uses the key concept of wireless sensor network and focuses on ECG signal. This system relies on 3 lead ECG clamp to capture real time ECG of the patient and GSM/GPRS SIM300 module for real time wireless transmission. . Since, we are dealing with an important parameter that is ECG data integrity is must and is accomplished using TCP protocol. The main processing element used is ARM7 and ECG signal is efficiently received on PC in MATLAB at doctor workstation.
\end{abstract}

Keywords: Wireless sensor network (WSN), 3 lead ECG clamp, Sim300, TCP protocol and ARM7.

\section{INTRODUCTION}

In many cases, Patient requires continuous monitoring and essentially needs lengthy stay in hospitals which is again becoming costly now days.

Traditional monitoring system allows continuous monitoring of vital parameters which require the sensors to be connected to bedside machines or PCs, and patient is essentially confined to bed. However, today's busy world and increase in sudden death events motivates for a monitoring system that continuously monitors remotely located patient.

Depending on this factor many researchers have developed patient monitoring system.

ECG Holter monitoring is the most widely used technique for providing ambulatory cardiac monitoring for capturing rhythm disturbances. A traditional Holter monitor can record up to 24 hours of ECG signals and the recorded data is subsequently retrieved and analyzed by a clinician. Due to the short duration involved and the unknown context within which

ECG signal is captured; reliable interpretation of the recorded data is always a challenge [1].

Telemetry is another way of monitoring remotely located patient. In such systems small distance transmission is achieved using coaxial cable, telephonic lines etc. Also for long distance communication, wireless telemetry using RF transmission technique is developed and M.Srinagesh, P.
Sarala, K.Durga Aparna proposed a system to improve quality of ECG signal in wireless telemetry [2][3].

However, current biomedical devices lack in the ability to provide large-scale analysis, simulations and computations at the patient's location. Wireless Sensor Network is becoming a promising technology for various applications. One of its potential deployments is in the form of Wireless Biomedical Sensor Network (WBSN) for measuring physiological signals. Many ECG monitoring system are developed based on WSN technology. Taking communication media into account many systems are developed that transmit ECG signal with wireless technologies like GSM and Zigbee efficiently. A system was developed to demonstrate efficient transmission of ECG signal to doctor workstation and no real time ECG signal was used [4].

Bluetooth has a wide variety of applications in the medical field. Scatternet is a technique used for transmission of ECG signal [5]. Real time ECG data can be transferred to the central location or to the mobile phones by compressing and using Bluetooth wireless technology [6]. Real time patient monitoring system using android smart phones is developed that it can be used to analyze the data and send alert messages to the patient if necessary [7][8]. Liang Kai, Xu Zhang, Yuan Wang, Huang Suibiao, Guan Ning, proposed a system where the ECG signals are captured using dry skin electrodes and then these signals extracted from the electrodes are amplified, filtered using band pass filter and then are transmitted using Bluetooth wireless technology [9]. 
Real time monitoring of remotely located patient is possible using wireless Zigbee technology [10][11]. Also when continuously monitoring a patient, streaming is one of the issue and is handled by using Linux OS [12]. With the use efficient wireless technology it is possible to transmit different data like temperature, pulse rate, ECG signal of different patients and also give warning signal with the help of GSM modem. Thus, multiple patients can be monitored with aid of technology [13][14][15][16][17]. However with this technique range is the main concern since Zigbee is having $100 \mathrm{~m}$ range. Also, another patient monitoring system was developed in which the remotely located patients are monitored continuously and meaningful data like heart beat, air flow etc are sent to the smart phones using GSM technology[18][19].

A new system based on cloud computing technology was developed. In this local server is used where patient's data is collected and is then transmitted to the doctor workstation with the help of Internet. Also to get the data from patient Zigbee wireless technology is used [20].

\section{PROPOSED SYSTEM}

In this proposed system, ARM7 with 3 lead ECG clamp and SIM300 module act as one sensor node. Figure 1 represents sensor node of the system.

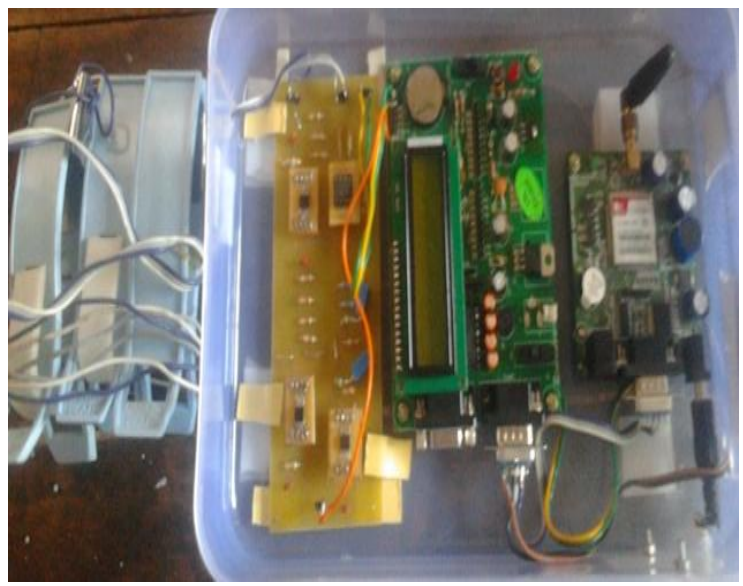

Fig1. Sensor node incorporating 3 lead ECG clamp, ARM7 and SIM300.

ECG signal is then captured using this 3 lead ECG clamp, signal is required to be processed so that it can be analyzed properly. Hence this signal is then given as input to the amplifier section consisting instrumentation amplifier and operational amplifier.

Once this signal is properly amplified then it is further processed using ADC of core processor ARM7 (LPC2148) and then the signal is transmitted with help of GPRS module SIM300 and TCP protocol. Finally at the doctor workstation signal is observed in MATLAB software with help of Internet

\section{HARDWARE IMPLEMENTATION}

In Amplifier and filter design, Output of left and right Arm clamps is fed as input to Instrumentation amplifier and the third clamp is grounded and is connected to right leg. Output of Instrumentation amplifier is then given to high pass filter which is set to $0.5 \mathrm{~Hz}$ in order to filter baseline noise. This filtered ECG acts as input to Notch filter that is set to $50 \mathrm{~Hz}$, implemented to eliminate powerline noise and finally the signal is fed to low pass filter that is set to $150 \mathrm{~Hz}$ since ECG data between $0.05 \mathrm{~Hz}-150 \mathrm{~Hz}$ is sufficient for proper diagnostics.

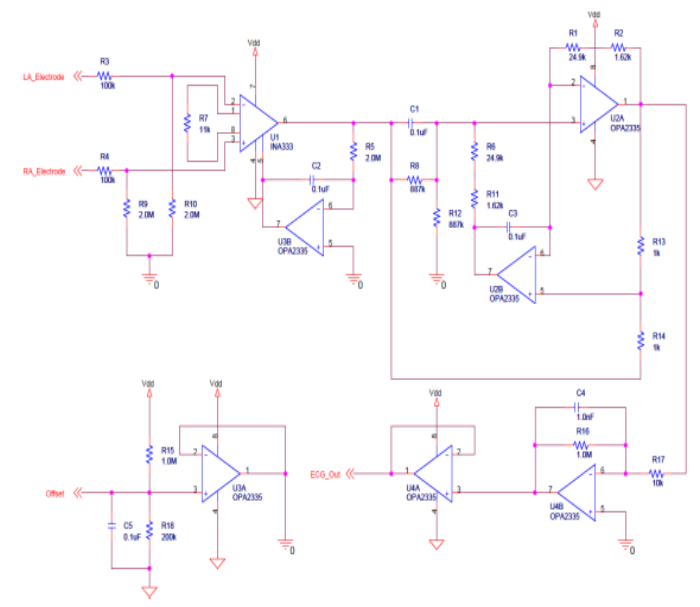

Fig 2 Amplifier and Filter design

\subsection{Hardware at Patient Side}

As can be seen from figure 4, hardware at patient side include, 3 lead ECG clamp, ECG front end system(amplification and processing circuit), ARM7, SIM300 and laptop to verify acquired signal and IP address.

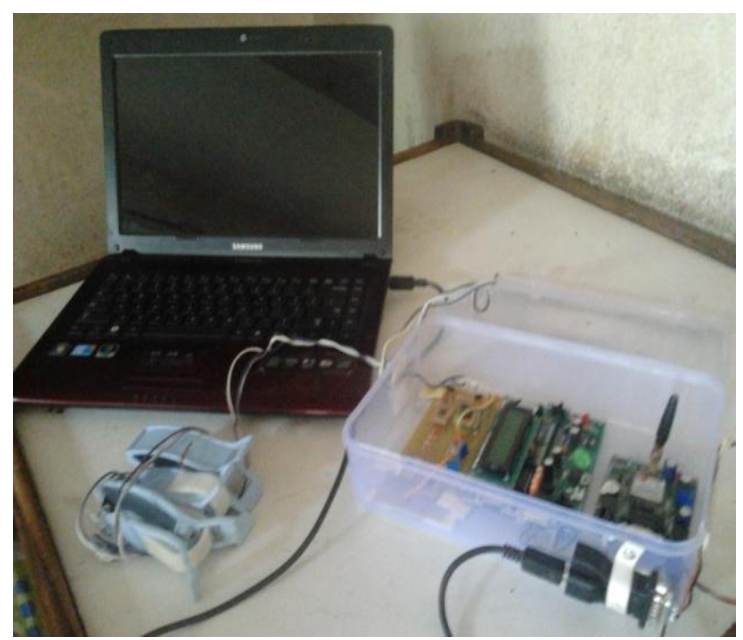

Fig 3 Hardware at Patient side 


\subsection{Hardware at Doctor Workstation}

The only requirement of hardware at doctor workstation is of a single PC or laptop having MATLAB 2013a installed and Internet connection.

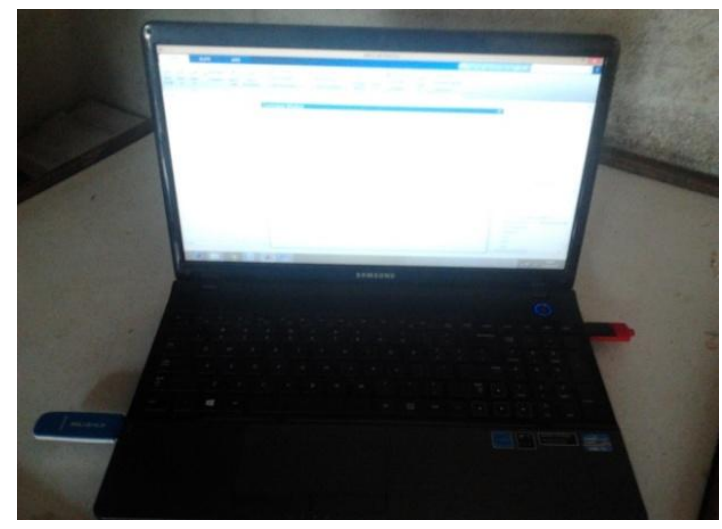

Fig 4 Hardware at Doctor Workstation

\section{SOFTWARE IMPLEMENTATION}

The ECG signal captured from 3 Lead ECG clamp is to be transmitted to the doctor workstation. For this the ECG signal is to be first digitized then should be sent to GPRS module through UART. Hence once the signal is captured ADC of ARM7 is initialized which is reference voltage $3.3 \mathrm{~V}$. This digitized signal is then given to UART0 of ARM7 Also, LCD is used to display word ECG. All this that is initialization of ADC, UART0 and LCD is done in Embedded C using Kiel $\mu$ vision4 (Snapshot shown in figure 4).

After getting the signal at UART0 the GPRS module transmits the signal to the doctor workstation. GPRS module is used in TCP mode and the same initialized using AT commands.

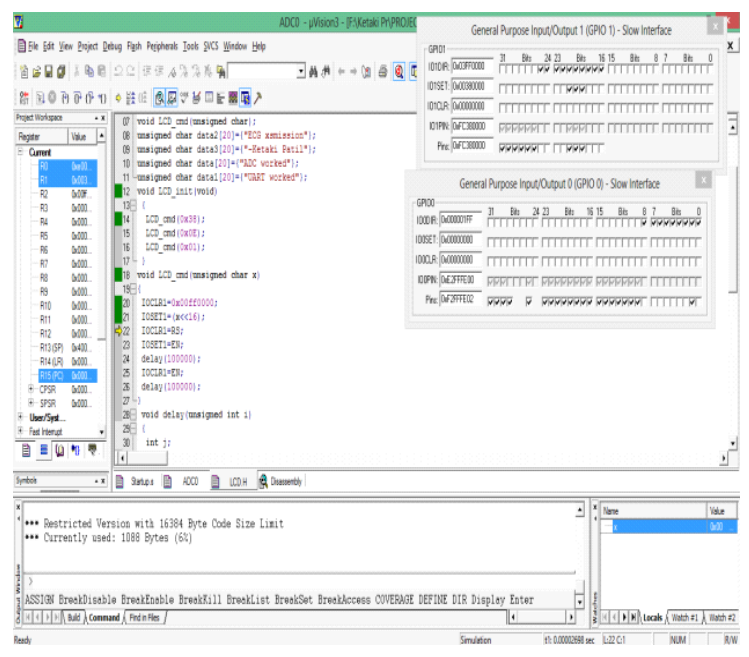

Fig 5 Snapshot of code and debugging of the same
Once the SIM 300 module is properly initialized using AT commands, it provides necessary IP address. That IP address is then put into MATLAB code (Figure 6) for TCP/IP. If this is properly done then, SIM300 detects remote IP address and provides message that connection is established properly and starts sending ECG signal continuously. This data is then observed in MATLAB 2013a.

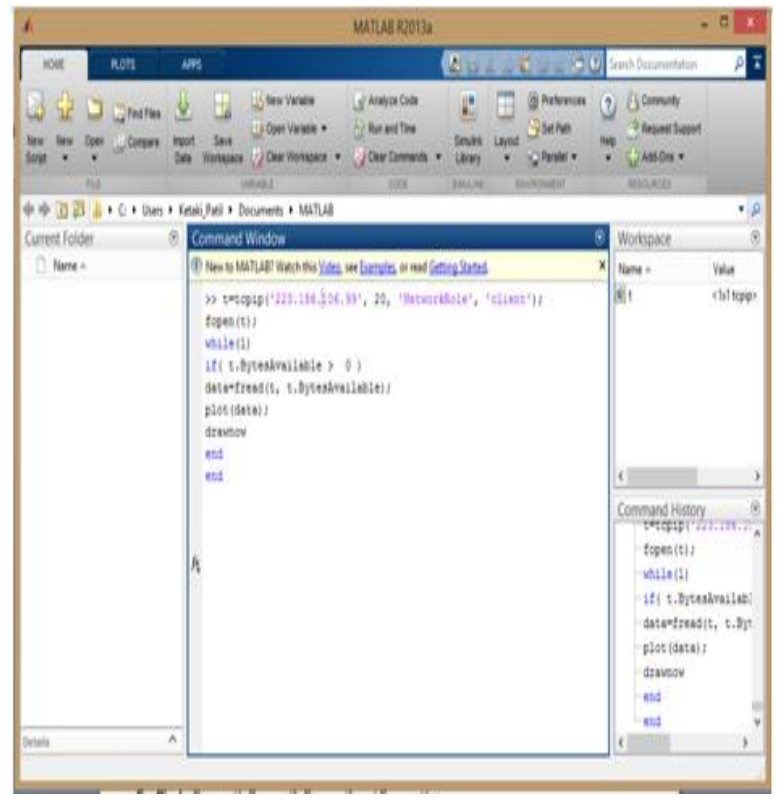

Fig 6 Snapshot of MATLAB code to observe ECG signal at doctor workstation

\section{RESULTS}

$>$ ECG signals vary from micro to milli volt range. In order to properly interpret the obtained data the signals acquired from the electrodes must be amplified.

$>$ In this system the gain is adjusted to 10 that is the signal must be amplified at least 10 times the original signal.

$>$ To verify the results that is whether the designed system provides amplified signal or not, DSO is used.

$>$ This DSO will provide the necessary amplitude and frequency of the signal from the output of the acquisition system (Figure7).

$>$ Further once the signal is properly amplified it is observed on PC.

$>$ The output in that case is seen in the Terminal software (Figure8).

$>$ Once the signal is satisfactorily observed at patient side it is transmitted to doctor workstation and is observed in MATLAB software (Figure 9). 


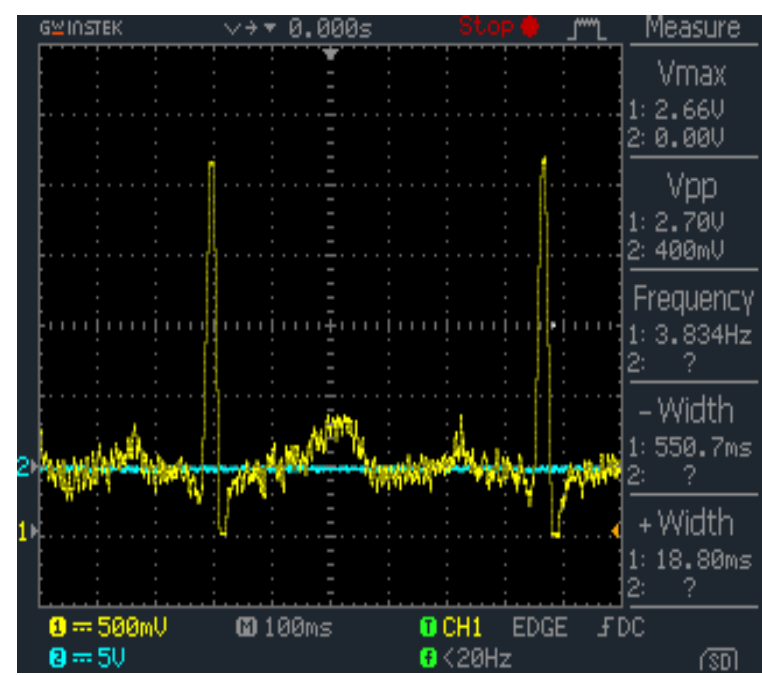

Fig 7 ECG signal captured on DSO

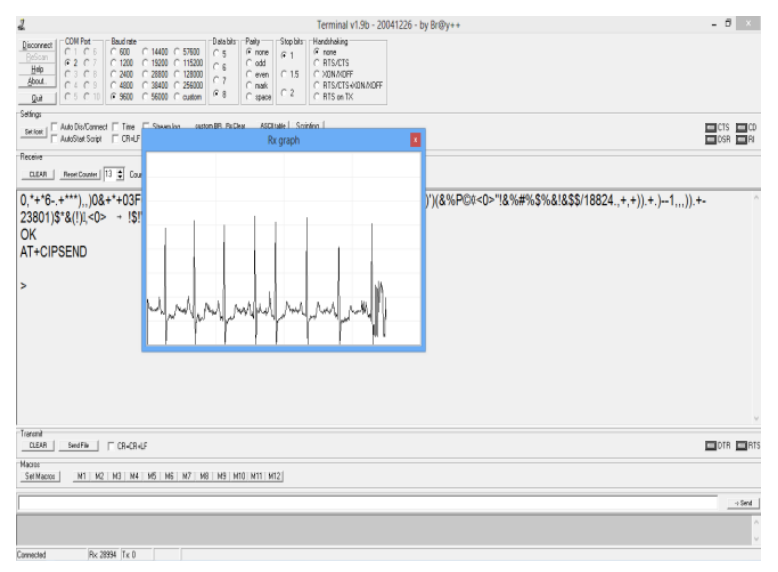

Fig 8 Snapshot of Real time ECG observed at patients side.

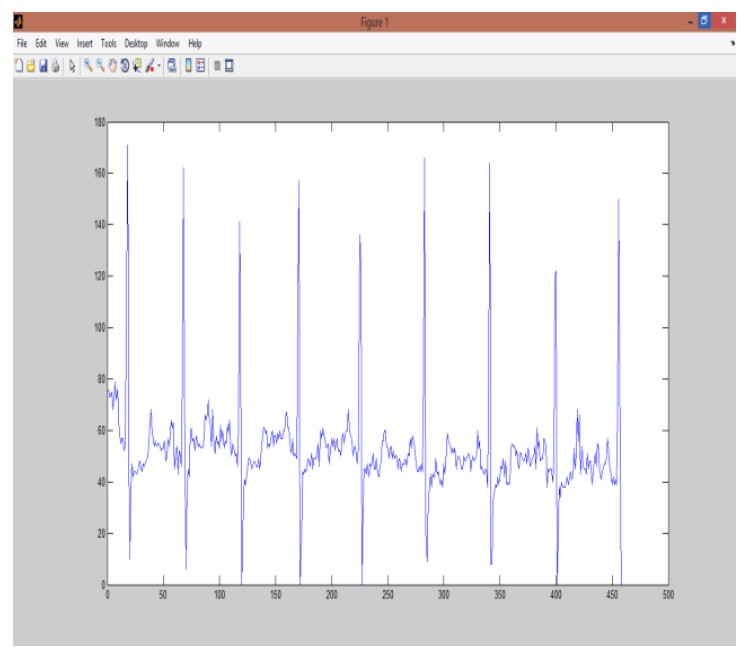

Fig 9 Snapshot of real time ECG observed at doctor workstation.

\section{CONCLUSIONS}

Real time ECG monitoring system for remotely located patient using ARM7 LPC2148 as core processor and MATLAB 2013a software at doctor workstation is developed. However since real time ECG is wirelessly transmitted there is high possibility of transmission noise or errors. But as we are observing signal in MATLAB, further processing of signal can be easily done in MATLAB Simulink and best signal can be achieved.

\section{REFERENCES}

[1] Role of cardiographer book.

[2] Document 120-08 "Telemetry Systems radio frequency" Handbook, Range commander's council.

[3] ECG Wireless Telemetry M.SRINAGESH, P. SARALA, K.DURGA APARNA, International Journal of Engineering and Innovative Technology (IJEIT) Volume 2, Issue 8, February 2013 ISSN: 2277-3754 ISO 9001:2008 Certified.

[4] N.M.Z. Hashim1, M. S. Sizali2 "Wireless Patient Monitoring System", International Journal of Science and Research (IJSR), India Online ISSN: 2319-7064".

[5] Sivaranjani a, Vignesh kumar k, Sathya s, Santhi b "Automatic patient monitoring system using scatternet for critical care", Journal of Theoretical and Applied Information Technology 15 June 2012. Vol. 40 No.1 ISSN: 1992-8645, E-ISSN: 1817-3195.

[6] Bo Yu, Liuqing Yang, Chia-chin chong "ECG Monitoring over Bluetooth: Data Compression and Transmission" IEEE conference on Wireless communication and networking ISSN: 1525-3511 (978-1-4244-6396-1) April 2010.

[7] Satish Patil, Pallavi Kulkarni "Ubiquitous Real Time ECG Monitoring System Using Android Smartphone" International Journal of Computer Science and Information Technologies, Vol. 4 (6) , 2013, 895 - 898, ISSN: 0975-9646.

[8] Byungkook Jeon, Jundong Lee and Jaehong Choi, "Design and Implementation of a Wearable ECG System" International Journal of Smart Home Vol. 7, No. 2, March, 2013.

[9] Liang Kai, Xu Zhang, Yuan Wang, Huang Suibiao, Guan Ning, "A system of portable ECG monitoring based on Bluetooth mobile phone" IEEE international Symposium on IT in medicine and education" Dec 2011.

[10] Rubina.A. Shaikh, Sajid Shaikh "Wireless Stand-alone Real Time Remote Patient Health Monitoring System" International Journal of Innovative Technology and Exploring Engineering (IJITEE) ISSN: 2278-3075, Volume-3, Issuse-7, December 2013.

[11] J. Yoo, L. Yan, S. Lee, Y. Kim, and H. J. Yoo, “A 5.2 $\mathrm{mW}$ self-configured wearable body sensor network controller and a $12 \mathrm{uW}$ wirelessly powered sensor for a 
continuous health monitoring system," IEEE J. SolidState Circuits, vol. 45, no. 1, pp. 178-188, Jan. 2010.

[12] Thanawattano C, Pongthonseri R, Dummin S, "Wearable wireless ECG sensor with cross-platform real-time monitoring" IEEE EMBS conference on biomedical Engineering and sciences" 978-1-46731664-4 Dec 2012.

[13] Enhancement of real-time multi-patient monitoring system based on wireless sensor networks, International Journal of Physical Sciences Vol. 6(4), pp. 664-670, 18 February, 2011.

[14] Remote Cardiac Arrhythmia Monitoring System Using Wireless Sensor Networks,11th International Conference on Development and Application Systems, Suceava, Romania, May 17-19, 2012

[15] Megalingam, RajeshKannan, Kaimal,D.M "Efficient Patient Monitoring for Multiple Patients Using WSN" IEEE international conference, Advances in mobile network, communication and network 978-1-46731869-3 Aug 2012.

[16] Real-time multi-patient monitoring system using arm and wireless sensor network, International Journal of Communication Network Security, ISSN: $2231-1882$, Volume-2, Issue-2, 2013.

[17] Firas Ibrahim "Remote Health Monitoring Systems for High Risk Patients" , Int. J. Open Problems Compt. Math., Vol. 5, No. 4, December 2012 ISSN 1998-6262; Copyright $\odot$ ICSRS Publication, 2012 www.i-csrs.org.

[18] Bhargavi Sundara, Dr. Kalyana Chakravarthi Sarvepalli, Sri Harsha Davuluri, "GSM BASED PATIENT MONITORING SYSTEM" IJRET: International Journal of Research in Engineering and Technology eISSN: 2319-1163 | pISSN: 2321-7308.

[19] Manita Rajput, Sadhana Pai, Umesh Mhapankar "Wireless Transmission of Biomedical Parameters Using GSM Technology" International Journal of Emerging Science and Engineering (IJESE) ISSN: 2319-6378, Volume-01, Issue-09, July 2013.

[20] R.Deepa, K.Boopathy "Cloud Care: A Remote Health Monitoring System” ISSN: 2319-5967 ISO 9001:2008 Certified International Journal of Engineering Science and Innovative Technology (IJESIT) Volume 3, Issue 1, January 2014. 